\title{
Skin prick testing with extensively heated milk or egg products helps predict the outcome of an oral food challenge: a retrospective analysis
}

Zein Faraj ${ }^{1}$ and Harold L Kim ${ }^{1,2^{*}}$

\begin{abstract}
Background: Cow's milk and hen's egg are the most frequently encountered food allergens in the pediatric population. Skin prick testing (SPT) with commercial extracts followed by an oral food challenge (OFC) are routinely performed in the diagnostic investigation of these children. Recent evidence suggests that milk-allergic and/or eggallergic individuals can often tolerate extensively heated (EH) forms of these foods. This study evaluated the predictive value of a negative SPT with EH milk or egg in determining whether a child would tolerate an OFC to the EH food product.
\end{abstract}

Methods: Charts from a single allergy clinic were reviewed for any patient with a negative SPT to EH milk or egg, prepared in the form of a muffin. Data collected included age, sex, symptoms of food allergy, co-morbidities and the success of the OFC to the muffin.

Results: Fifty-eight patients had negative SPTs to the EH milk or egg in a muffin and underwent OFC to the appropriate EH food in the outpatient clinic. Fifty-five of these patients tolerated the OFC. The negative predictive value for the SPT with the EH food product was $94.8 \%$.

Conclusions: SPT with EH milk or egg products was predictive of a successful OFC to the same food. Larger prospective studies are required to substantiate these findings.

\section{Background}

Although estimates of prevalence are heterogeneous in medical literature, cow's milk and hen's egg are consistently reported as two of the most common food allergens in the pediatric population [1]. The diagnostic investigation for food allergy commences with skin prick testing (SPT) with commercial extracts of suspected allergens. In cases of true IgE-mediated allergic reactions, a localized cutaneous swelling in the form of a 'wheal' usually ensues. Typically, negative SPTs are followed by an oral food challenge (OFC), the gold standard, to definitively rule out food allergy.

The standard management of food allergy is strict avoidance of the confirmed allergen [2]. For both milkallergic and egg-allergic patients, this restriction limits

\footnotetext{
* Correspondence: hlkimkw@gmail.com

'Michael G. DeGroote School of Medicine, McMaster University, Hamilton, ON, Canada

${ }^{2}$ Department of Medicine, University of Western Ontario, London, ON,
} Canada

(C) 2012 Faraj and Kim; licensee BioMed Central Ltd. This is an Open Access article distributed under the terms of the Creative Commons Attribution License (http://creativecommons.org/licenses/by/2.0), which permits unrestricted use, distribution, and reproduction in any medium, provided the original work is properly cited. dietary options. Undoubtedly, adherence to this regimen can be burdensome, limits dietary variety and negatively impacts quality of life.

In recent years, evidence has emerged suggesting that the majority of children with milk and/or egg allergy can tolerate these foods when they are extensively heated $(\mathrm{EH})[3,4]$. Extensive heating alters the allergenic proteins to which IgE antibodies typically form and allergenicity is attenuated in cases of certain allergens such as milk and egg [5]. Furthermore, it has been suggested that exposure may be therapeutic and extended delay in introduction may be detrimental by increasing risk of allergy and delaying the development of tolerance [6]. It has also been proposed that development of tolerance to $\mathrm{EH}$ products precedes tolerance to the unheated product by several years [7]. These findings imply that milk-allergic and egg-allergic patients' diets may have been unnecessarily restricted. Nonetheless, a subset of these children are truly allergic to both $\mathrm{EH}$ and non-EH milk and/or eggs, and will react to both forms in 
oral challenges. This may be due to the presence of heat-stable proteins that can maintain their allergenicity despite extensive heating [5]. It is important to consider whether the advantage of potentially being able to consume EH products is worth the risk taken during the OFC. For example, one study reported that $73 \%$ of eggreactive children who had a positive SPT to commercial extracts were able to tolerate egg baked in a muffin and cooked in a waffle during a physician-supervised OFC [4]. The subjects who did not tolerate the EH egg were at risk of experiencing an anaphylactic reaction.

This study aimed to evaluate whether a negative fresh food SPT with the EH milk or egg products serves as a reliable marker in predicting tolerance to an OFC with the same product in the outpatient clinical setting.

\section{Methods}

A retrospective chart review was performed on all patients undergoing cow's milk or hen's egg skin prick testing at a single allergy and immunology clinic in Kitchener, Ontario during a 2 year time period from 20092011. Patients were deemed eligible if they were between the ages of 6 months and 18 years at the time of an initial positive SPT to cow's milk and/or hen's egg commercial extracts, had a subsequent negative SPT to the $\mathrm{EH}$ version of the allergen, and proceeded to an OFC with the EH milk or egg product. An SPT was considered positive if the wheal's diameter was at least three millimeters larger than the negative control test. All eligible subjects had either previously experienced an allergic reaction to milk or egg or had a positive SPT predictive of an allergy. Subjects with a history of reaction to baked milk or egg products were excluded from the study.

The anterior surface of the forearm was used for skin prick testing with commercially-prepared cow's milk and hen's egg extract testing solutions (Omega Labarotories Limited). A drop of egg or milk extract, a negative control, and a positive histamine control were applied to the forearm. The drops were pricked using a Hollister-Stier lancetter and the tests were read after 15 minutes.

Later, SPT was performed with fresh food extracts prepared from an EH milk or egg product. The EH product consistently used was a wheat-based muffin baked with either one third of an egg (both egg yolk \& white) per muffin at $350^{\circ} \mathrm{F}$ for 30 minutes or $40 \mathrm{~mL}$ of homogenized milk per muffin at $350^{\circ} \mathrm{F}$ for 30 minutes. These amounts were deemed greater than would be found in the ingredients of an average baked good of a similar serving size. The muffins were prepared at home by the caregivers and brought to the appointment. Approximately 1 gram of the muffin was thoroughly mixed with $10 \mathrm{ml}$ of water using a tongue depressor. A drop of the slurry was placed on the forearm and pricked with a Hollister-Stier lancetter. The tests were read after 15 minutes.

The OFC was performed in 30-minute intervals, with administration of each dose only if the preceding portion was tolerated. Initially $10 \%$ of the muffin was administered for ingestion, followed by three portions of $30 \%$. Subjects were monitored in the clinic for 60 minutes following the OFC.

The SPT and OFC for all subjects were performed under direct supervision of an allergist in his outpatient clinic.

\section{Results}

Of the 128 subjects found to have a positive SPT to unheated cow's milk or hen's egg, 58 subjects (median age 3.5 years; range $1.25-13$ years) met the remainder of the inclusion criteria for this study. Fourteen were milkallergic whereas 40 were egg-allergic (Figures 1 and 2). The median age at first reaction was 1 year (range 0.57 years). Initial allergic reaction varied but included cutaneous symptoms such as hives, pruritis or flushing (84.5\%), upper airway symptoms including sneezing or throat symptoms (10.3\%), lower airway symptoms including wheeze-bronchospasm or respiratory distress (3.5\%), gastrointestinal symptoms such as nausea, vomiting, cramping abdominal pain, bloating, diarrhea (22.4\%), and cardiovascular symptoms evidenced by dizziness or hypotension (3.5\%). Anaphylaxis was reported in $8.6 \%$ of patients at initial reaction (Table 1 ).

Other atopic conditions were highly prevalent in children with cow's milk and hen's egg allergies. Co-morbid asthma was found in $31.0 \%$ of subjects, allergic rhinitis in $29.3 \%$, and history of current or resolved atopic dermatitis in $51.7 \%$. A positive family history of atopy was reported in $74.1 \%$ of the subjects and multiple food allergies reported in $79.3 \%$ (Table 1).

The median wheal size for initial SPT testing with unheated milk or egg products was $5 \mathrm{~mm}$ (range 3 $9 \mathrm{~mm}$ ). All the subsequent fresh SPTs with the EH milk or egg products were negative as per the inclusion criteria. This was followed by an OFC with the same food. Fifty-five children (94.8\%) did not experience any allergic reaction to EH milk or eggs. Three children (5.2\%)

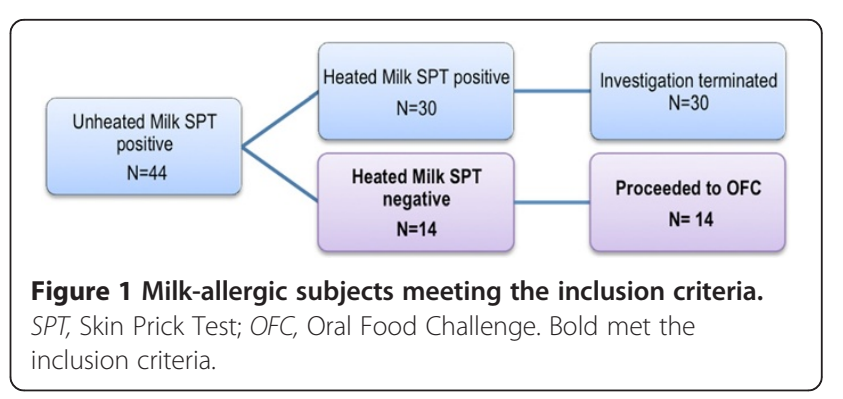




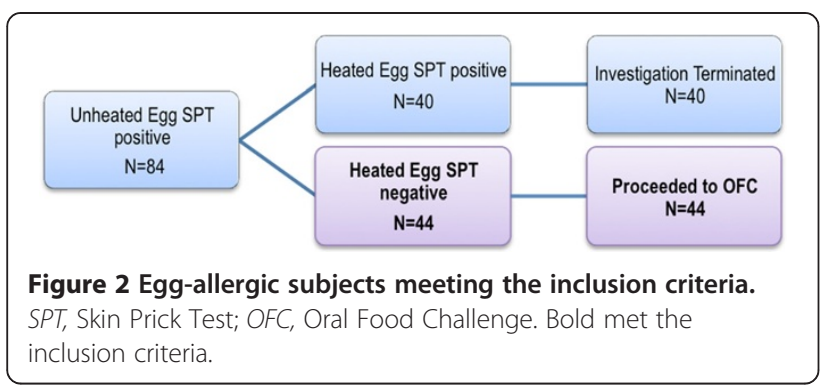

reacted to the $\mathrm{EH}$ product and were therefore allergic to the food in both forms. All reactions to OFC occurred in subjects from the egg-allergic cohort (Figure 3).

Only one of the three reactions was anaphylactic in nature, as defined by the clinical criteria from the second symposium on anaphylaxis [8]. This 2.25 year old patient experienced eye swelling after $40 \%$ of the muffin and was given a dose of diphenhydramine. Fifteen minutes later, symptoms worsened to include vomiting. An hour later, the patient became dyspneic and drowsy. While oxygen saturation level fell to $93 \%$ on pulse oximetry, he maintained a pulse of 100-125 and lungs remained clear to auscultation. Epinephrine was administered twice. This was the child's first exposure to egg and therefore there was no previous history of an allergic reaction to

Table 1 Characteristics, history and symptoms of subjects included in the study, reported both as a summative total and as milk-allergic or egg-allergic independently

\begin{tabular}{llll}
\hline Characteristic & All subjects & $\begin{array}{l}\text { Milk-Allergic } \\
\text { only }\end{array}$ & $\begin{array}{l}\text { Egg-Allergic } \\
\text { only }\end{array}$ \\
\hline Total subjects (\%) & $58(100 \%)$ & $14(24.1 \%)$ & $44(75.9 \%)$ \\
Male sex (\%) & $34(59 \%)$ & $12(86 \%)$ & $22(50 \%)$ \\
$\begin{array}{l}\text { Age (y), median } \\
\text { (range) }\end{array}$ & $3.5(1.25-13)$ & $3.5(2-9)$ & $3.5(1.25-13)$ \\
Co-morbidities (\%) & & & \\
$\quad$ Asthma & $18(31.0 \%)$ & $6(42.9 \%)$ & $12(27.3 \%)$ \\
$\quad$ Allergic Rhinitis & $17(29.3 \%)$ & $4(28.6 \%)$ & $13(29.6 \%)$ \\
$\quad$ Atopic Dermatitis & $30(51.7 \%)$ & $7(50 \%)$ & $23(52.3 \%)$ \\
$\quad$ Other food allergy & $49(79.3 \%)$ & $12(85.7 \%)$ & $34(77.3 \%)$ \\
$\begin{array}{l}\text { Family history of } \\
\text { atopy }\end{array}$ & $43(74.1 \%)$ & $11(78.6 \%)$ & $32(72.7 \%)$ \\
Age at first reaction & $1(0.5-7)$ & $0.5(0.5-1.25)$ & $1(0.5-7)$ \\
(y), median (range) & & & \\
$\begin{array}{l}\text { Symptoms at first } \\
\text { reaction (\%): }\end{array}$ & & & $3(2.3 \%)$ \\
$\begin{array}{l}\text { Cutaneous } \\
\text { Upper Respiratory }\end{array}$ & $6(10.3 \%)$ & $3(21.4 \%)$ & $3(6.8 \%)$ \\
$\begin{array}{l}\text { Lower Respiratory } \\
\text { Gastrointestinal }\end{array}$ & $2(3.5 \%)$ & $2(14.3 \%)$ & $0(0 \%)$ \\
$\begin{array}{l}\text { Cardiovascular } \\
\text { Anaphylaxis }\end{array}$ & $2(3.5 \%)$ & $2(14.3 \%)$ & $0(0 \%)$ \\
\hline
\end{tabular}

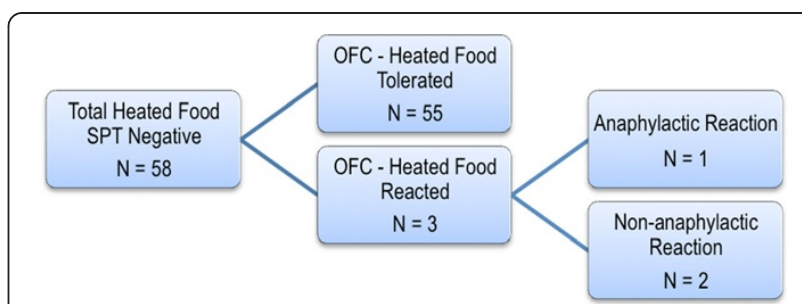

Figure 3 Summative results of both milk and egg allergic subjects to an OFC, including anaphylactic reactions. SPT, Skin Prick Test; OFC, Oral Food Challenge.

egg. The wheal diameter of his SPT to commercial hen's egg was mild at $3 \mathrm{~mm}$.

The two non-anaphylactic reactions were in children aged 2.5 and 1.75 respectively. The first of the two nonanaphylactic patients developed hives, followed by pallor and dizziness but maintained stable vital signs after $40 \%$ of the muffin. Epinephrine was also administered, and his symptoms resolved within 15 minutes. Previous history of anaphylaxis was documented in this patient, with symptoms of a rash, throat tightening and vomiting. His positive SPT to commercial hen's egg was recorded at $5 \mathrm{~mm}$. The second patient developed mild peri-oral erythema after consuming one tenth of the muffin, and no medical interventions were necessary; the challenge was terminated and his symptoms resolved. The history of his initial reaction to egg was limited to a mild rash, and his positive SPT to commercial hen's egg was mild at $3 \mathrm{~mm}$.

\section{Discussion}

The vast majority of subjects with a negative fresh food SPT of the EH food product had successful OFCs to the baked muffins. Fifty-five of the 58 patients tolerated the oral challenge and were encouraged to re-introduce baked eggs or milk into their diets, whereas three reacted and were assumed to be allergic to the food products in all forms. Only one of the three had an anaphylactic reaction (Figure 3). The negative predictive value for the SPT with the extensively heated food product was $94.8 \%$.

This study is the first to propose performing a fresh food SPT in children with milk and egg food allergies in order to predict outcomes of an OFC with EH food. This may serve as a practical marker for children likely to be tolerant of EH milk and egg OFCs. Although previous studies have shown that the majority of food-allergic children tolerate the same foods in their $\mathrm{EH}$ forms, the proportion of children experiencing an allergic reaction during the OFC remained significant. Reaction rates of $23 \%$ were reported by two separate studies, one challenging milk-allergic children to an OFC with $\mathrm{EH}$ milk and the second challenging egg-allergic children to an OFC 
with EH egg products. Twenty-three out of a sample size of 100 reacted in the milk allergy study, whereas 27 out of 117 reacted in the egg allergy study [3,4]. In our study, the risk of reaction during the diagnostic process was reduced from $23 \%$ to $5 \%$ by performing an SPT with $\mathrm{EH}$ foods prior to their OFC.

Many studies have explored the utility of serologic testing in both the diagnosis of allergy as well as ability to predict tolerance to EH products. For example, specific IgE antibodies to the egg protein ovomucoid have been suggested to be predictors of reactivity to EH egg products, and clinical decision points have been proposed [9]. A recent study suggests a role for the specific IgE/ $\mathrm{IgG}_{4}$ antibodies to ovalbumin (OVA) and ovomucoid (OVM) in predicting reactivity to extensively heated egg products in egg-allergic children, however this is not a clinically practical or available option [10]. Neither food-specific IgE levels nor SPT responses to commercial food extracts are entirely reliable in identifying children likely to be tolerant of EH milk and egg products [11]. Our proposal to incorporate fresh food SPT in the diagnostic evaluation of milk and egg allergic children may serve as a practical, easy alternative in an allergist's clinic.

Nevertheless, this study has some limitations. There are inherent flaws in any retrospective study, including lack of blinding of any party. While physician notes from patient encounters were detailed, the data were not originally collected for the purpose of research. Furthermore, the sample size was small, non-homogeneous for one food allergy, and had a much larger egg-allergic cohort. Baseline characteristics between milk and egg allergic individuals were not entirely balanced, and it is unclear whether this is due to a true difference between the two populations. Future studies should assess milk and egg food allergies separately. Although parents were given specific instructions with regards to how to bake the muffins, this factor was not otherwise controlled. Moreover, data were insufficient for calculations of positive predictive value, specificity and sensitivity. Lastly, this study was performed at one centre with the observations and clinical judgment of one clinician; larger multi-clinician, multi-centre trials would be able to better substantiate our findings.

\section{Conclusions}

The majority of patients with milk or egg allergy who had negative SPTs with the EH milk or egg products respectively tolerated the EH form of the food in an OFC. Based on the data collected in this study, skin prick testing with $\mathrm{EH}$ food carries a negative predictive value of $94.8 \%$ and may be a reliable marker for identifying children likely to tolerate EH milk or egg.

\section{Abbreviations}

SPT: Skin prick test; OFC: Oral food challenge; EH: Extensively heated.

\section{Competing interests}

The authors declare that they have no competing interests.

\section{Acknowledgements}

None to declare.

\section{Authors' contributions}

ZF helped design the study, conducted the chart review for data collection, analyzed the data and wrote the paper. HK conceived the study and was the primary clinician performing all tests and challenges on subjects. Both authors read and approved the final manuscript.

\section{Authors' information}

Zein Faraj is a senior medical student at McMaster University, an MD Candidate in the class of 2012. She has previously completed Bachelor of Health Sciences (Honours) degree at McMaster University.

Dr. Harold Kim is an Allergist and Clinical Immunologist practicing in Kitchener, ON. He is an Adjunct Professor at the University of Western Ontario and an Assistant Clinical Professor at McMaster University.

Received: 11 January 2012 Accepted: 16 May 2012

Published: 16 May 2012

\section{References}

1. Rona RJ, Keil T, Summers C, Gislason D, Zuidmeer L, Sodergren E, Sigurdardottir ST, Lindner T, Goldhahn K, Dahlstrom J, McBride D: The Prevalence Of Food Allergy: A Meta-analysis. J Allergy Clin Immunol 2007, $120 v(3): 638-646$.

2. American College of Allergy, Asthma \& Immunology: Food allergy: a practice parameter. Ann Allergy Asthma Immunol 2006, 96(3 Suppl 2):S1-S68.

3. Nowak-Wegrzyn A, Bloom K, Sicherer SH, Shreffler WG, Noone S, Wanich N, Sampson HA: Tolerance To Extensively heated Milk In Children With Cow's Milk Allergy. J Allergy Clin Immunol 2008, 122(2):342-347.

4. Lemon-Mule H, Sampson HA, Sicherer SH, Shreffler WG, Noone S, NowakWegrzyn A: Immunologic Changes In Children With Egg Allergy Ingesting Extensively Heated Egg. J Allergy Clin Immunol 2008, 122(5):977-983.

5. Konstantinou GN, Kim JS: Paradigm shift in the management of milk and egg allergy: baked milk and egg diet. Immunol Allergy Clin N Am 2012, 32:151-164.

6. Kim JS, Sicherer S: Should avoidance of foods be strict in prevention and treatment of food allergy? Curr Opin Allergy Clin Immunol 2010, 10:252-257.

7. Clark AT, Skypala I, Leech SC, Ewan PW, Dugue P, Brathwaite N, Huber PAJ, Nasser SM: British Society for Allergy and Clinical Immunology guidelines for the management of egg allergy. Clinical and Experimental Allergy 2010, 40:1116-1129.

8. Sampson HA, Munoz-Furlong A, Campbell RL, Adkinson NF, Bock SA, Branum A, et al: Second Symposium On The Definition And Management Of Anaphylaxis: Summary Report - Second National Institute Of Allergy And Infectious Disease/Food Allergy And Anaphylaxis Network Symposium. J Allergy Clin Immunol 2006, 117:391-397.

9. Ando H, Moverare R, Kondo $Y$, Tsuge I, Tanaka A, Borres MP, Urisu A: Utility of ovomucoid-specific lgE concentrations in predicting symptomatic egg allergy. J Allergy Clin Immunol 2008, 122:584-588.

10. Caubet JC, Bencharitiwong R, Moshier E, Godbold JH, Sampson HA, NowakWegrzyn A: Significance of ovomucoid- and ovalbumin-specific $\lg E / \operatorname{lgG}_{4}$ ratios in egg allergy. J Allergy Clin Immunol 2012, 129:739-747.

11. Nowak-Wegrzyn A, Sampson HA: Future therapies for food allergies. J Allergy Clin Immunol 2011, 127:558-573.

\section{doi:10.1186/1710-1492-8-5}

Cite this article as: Faraj and Kim: Skin prick testing with extensively heated milk or egg products helps predict the outcome of an oral food challenge: a retrospective analysis. Allergy, Asthma \& Clinical Immunology 2012 8:5. 